\title{
CONSIDERACIONES EN TORNO A LA FIGURA DEL SEguNdo JEFE
}

Por LUIS BLANCO DE TELLA

65.012 .4

Sumario: 1. Introducción : 1,1. Definición y requisitos. 1,2. Tendencias generales.2. Variedades orgánicas de la figura del segundo jefe: 2,1 . Segundas jefaturas en sentido propio. 2,2. Segundas jefaturas impropias.

\section{Introducción}

-N distintos niveles de las organizaciones públicas y privadas se puede observar frecuentemente la existencia de órganos permanentes calificados con carácter formal como segundas jefaturas. La nomenclatura aplicada a los citados órganos es, en la práctica, muy variable y escasamente indicativa de su naturaleza y contenido funcional.

En efecto, aunque normalmente las citadas posiciones suelen designarse anteponiendo las partículas sub o vice a la denominación del organo o puesto inmediatamente superior, existen segundas jefaturas cuya denominación no revela en absoluto tal carácter. Así, por ejemplo, la figura del secretario general aparece en ciertas uni- 
DA-1967, núm. 109. LUIS BLANCO DE TELLA. Consideraciones en torno a la figura del segundo ...

dades con todos los requisitos y atributos proplos de una segunda jefatura en sentido formal. $Y$ del mismo modo, como veremos más adelante, puede llegarse a la conclusión de que ciertas organizaciones y dependencias administrativas, a pesar de contar con un órgano al que se asigna la denominación de segundo jefe, carecen en realidad de tal figura.

Existe en este punto, por tanto, un problema terminológico que se presta a oscurecer el alcance de los conceptos y que requiere, en consecuencia, una previa aclaración.

\section{1,1 DEFINICIóN Y REQUISITOS}

Dejando aparte las situaciones de puro hecho en las que un determinado individuo asume la posición de segundo jefe al margen del esquema formal, y limitando nuestras observaciones al examen de dicho esquema, creemos que puede considerarse como segunda jefatura toda posición permanente $y$ única en su rango dentro de la organización o de un sector o dependencia determinada, que en la situación normal de presencia del primer jefe tiene atribuidas formalmente funciones de dirección más o menos amplias sobre todos o sobre alguno de los elementos orgánicos integrados en el escalón inmediato inferior.

De esta especie de definición se deduce que, a nuestro juicio, los requisitos que necesariamente han de concurrir en la figura que se examina son:

- Posición de carácter regular y permanente.

$\rightarrow$ Posición únlca en rango dentro de la unidad de que se trate.

- Posición cuyo contenido propio incluye funciones de dirección más o menos extensas (independientemente de las que puedan corresponder al titular en el supuesto de sustitución del inmediato superior).

De todo ello se desprende que no consideramos requisitos esenciales de la figura del segundo jefe, aunque concurran normalmente en la misma, las siguientes circunstancias:

- El llamamiento a la sustitución del superior inmediato (determinadas segundas jefaturas que poseen indiscutiblemente este carácter no son llamadas a ocupar el puesto del superior).

- El ejercicio de funciones directivas por vía de delegación (la delegación en sentido propio afecta a la dinámica de la organización, no a su estructura formal).

- La facultad de representar al superior. 
DA-1967, núm. 109. LUIS BLANCO DE TELLA. Consideraciones en torno a la figura del segundo ...

Si se acepta la delimitación que hemos propuesto en la anterior definición resulta que, como primera consecuencia, podremos hablar en lo sucesivo de segundas jefaturas propias e impropias (aquéllas en que falta algún requisito esencial o en las que sólo se registran circunstancias accesorias). A su vez, dentro de cada uno de ambos grupos, podrán ser aisladas y caracterizadas distintas variedades orgánicas de existencia formal, todas ellas frecuentes en la práctica.

Antes, sin embargo, creemos conveniente justificar la importancia del problema llamando la atención sobre el desarrollo actual $\rightarrow$ y posiblemente abuso-de tales figuras.

\section{1,2 Tendencias Generales}

Es evidente que existe una acusada tendencia a multiplicar las segundas jefaturas. Organismos o unidades administrativas cuyo esquema inicial carece de la repetida figura adoptan, al cabo de poco tiempo y muchas veces por vía de imitación, las disposiciones necesarias para establecerla en distintos niveles orgánicos comenzando normalmente por los superiores e intermedios y llegando, en ocasiones, hasta los últimos (subjefe de servicio, de sección e incluso de negociado). Los argumentos con que trata de justificarse la creación de tales posiciones suelen referirse normalmente a las circunstancias que antes hemos calificado de accesorias:

- La necesidad de asegurar la continuidad en el ejercicio de las funciones directivas dotando al primer jefe de un sustituto.

- La conveniencia de liberar al jefe de una parte de su trabajo haciendo posible la delegación de ciertas funciones que le impiden desempeñar satisfactoriamente aquellas otras consideradas de mayor interés.

- La conveniencia de asegurar la representación del superior en aquellas circunstancias y ocasiones en que no es posible su asistencia personal.

Estas consideraciones, de uso frecuente en los preámbulos de las disposiciones de creación de dichas figuras, no son suficientes, en nuestra opinión, para justificar con carácter general la existencia de las mismas.

En efecto, la indiscutible necesidad de garantizar la continuidad de las funciones directivas en un determinado nivel puede ser atendida satisfactoriamente mediante el establecimiento de un orden expreso de llamamientos a la sustitución, entre los subordinados del escalón inmediato inferior. Del mismo modo, la ausencia de segun- 
DA-1967, núm. 109. LUIS BLANCO DE TELLA. Consideraciones en torno a la figura del segundo ...

da jefatura no supone siempre obstáculo para delegar de forma adecuada las funciones que indebidamente recargan al órgano de que se trate ni hace imposible la representación de aquél.

Por todo ello, un juicio de primera mano acerca de la figura del segundo jefe en las organizaciones administrativas no puede afirmar, en principio, su absoluta necesidad. En nuestra opinión, ha de estimarse, por el contrario, que la creación de segundas jefaturas, especialmente en niveles intermedios e inferiores, constituye muchas veces una práctica viciosa, un procedimiento para alargar la escala jerárquica y una forma de aumentar los costes de la organización.

Coincidiendo con este punto de vista se ha dicho muy gráficamente, aunque sin llegar a generalizaciones radicales, que «en nueve casos de cada diez el segundo jefe es una quinta rueda» (1).

La experiencia demuestra cumplidamente, en efecto, que determinadas organizaciones, cuya eficiencia no deja lugar a dudas de ningún género, no han contado nunca con una segunda jefatura. Este hecho puede constatarse sin dificultad en ciertos organismos y dependencias de nuestra Administración pública.

Pero al lado de estos ejemplos, y contrastando con ellos, resulta igualmente cierto que en otros casos el segundo jefe juega en la práctica un papel fundamental, llegando en ocasiones hasta el punto de constituir la pieza maestra en el funcionamiento de la organización. No obstante, en tales casos, la segunda jefatura suele representar, a nuestro modo de ver, un remedio orgánico adoptado para corregir determinadas anomalias estructurales que impiden el ejercicio satisfactorio de las funciones directivas (exagerada amplitud de la esfera de control, heterogeneidad del contenido funcional del organismo o unidad, acumulación de funciones no directivas en el vértice, etc.). Esto equivale a decir que, en la mayoría de las ocaslones, una estructura racional o un aligeramiento de cometidos asignados al.jefe superior puede hacer innecesaria la segunda jefatura.

En definitiva, estimamos que las segundas jefaturas pueden considerarse justificadas solamente en supuestos excepcionales y en ciertos niveles en los que las consideraciones de tipo politico deben predominar sobre las de orden estrictamente administrativo. Por lo demás, la proliferación de tales órganos y puestos revela en muchos casos un ejercicio de la función de organizar ajustado a consideraciones personales más bien que a razones o necesidades objetivas.

(1) Malone, Philip. Conferencias desarroliadas durante el II Curso de Diplomados de $O$. y $M$. en la Escuela Nacional de Administración Pública, 1963. 
DA-1967, núm. 109. LUIS BLANCO DE TELLA. Consideraciones en torno a la figura del segundo ...

\section{Variedades orgánicas de la figura del segundo jefe}

El contenido asignado formalmente a las wegundas jefaturas varia considerablemente. En algunas ocasiones dicha figura aparece regulada con tal oscuridad e imprecisión que, aparte del llamamiento a la sustitución del superior, sólo se le asignan «aquellas funciones que se le deleguen o encomienden expresamente». En tales supuestos la posición orgánica de la segunda jefatura, salvo en lo relativo a su rango, es tan imprecisa que escapa a toda calificación que se pretenda realizar aplicando los conceptos acuñados por la teoría de la organización.

No es posible, en efecto, determinar en muchos casos si la segunda jefatura constituye o no un nivel orgánico más o si queda al margen de la línea de autoridad-responsabilidad. La posición real que en tales supuestos asuma la segunda jefatura en la dinámica de la organización dependerá de la amplitud y naturaleza de las delegaciones que el jefe superior le confiera o del ejercicio de autoridad de hecho en mayor o menor medida. Dichos casos de indeterminación, como todos los de fluidez orgánica en general, no admiten representación gráfica alguna ni pueden ser calificados, con arreglo a nuestra anterior definición, como segundas jefaturas propias ni impropias, razón por la cual quedan al margen de las consideraciones siguientes.

\section{2,1 Segundas Jefaturas en SEntido PROpio}

Todas ellas se caracterizan por la interposición formal más o menos intensa en la linea de autoridad-responsabilidad como situación permanente. Dentro del conjunto de figuras orgánicas que poseen este requisito cabe separar las siguientes variedades:

\section{2,11 Interposición total}

En este supuesto, el más radical y absoluto, el segundo jefe constituye, a todos los efectos, un nivel orgánico interpuesto entre el jefe superior y todas las unidades o puestos del escalón subordinado (figura 1). Dichos elementos, en el esquema oficial de la organización, aparecen vinculados directa y exclusivamente a la segunda jefatura, aunque, como es natural, pueden mantener contactos de hecho más o menos frecuentes con el jefe superior. 
DA-1967, núm. 109. LUIS BLANCO DE TELLA. Consideraciones en torno a la figura del segundo ...

La segunda jefatura, en este primer supuesto, no resuelve ningún problema de naturaleza directiva. Simplemente produce su desplazamiento. No debe olvidarse que, con independencia de otras obligaciones profesionales asignadas a veces en medida excesiva a las posiciones de jefatura, las funciones directivas abarcan únicamente aquella parte de la actividad del dirigente encaminada a asegurar

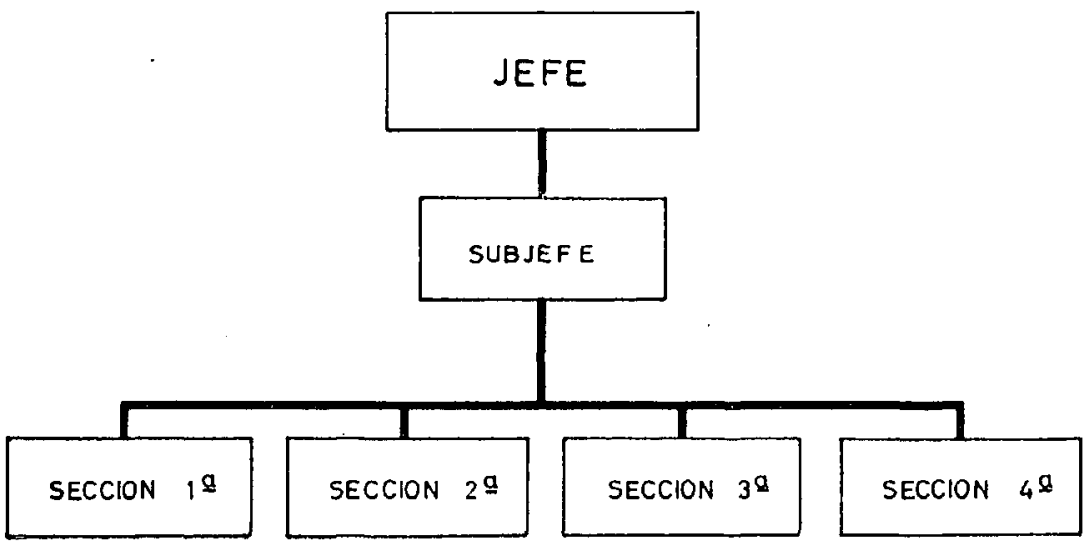

Figura 1

la cooperación de los miembros o elementos del grupo subordinado y, en consecuencia,. se proyectan hacia abajo.

Una amplia delegación de atribuciones, en la variedad que se examina, libera en gran medida al jefe superior, permitiéndole su proyección hacia arriba o hacia el exterior de la organización. En líneas generales esta modalidad sólo puede resultar aconsejable en algún supuesto excepcional.

\section{2,12 Interposición parcial}

En este supuesto - que en realidad puede abarcar distintas variantes- los elementos del escalón subordinado dependen simultáneamente de las dos jefaturas superpuestas. La linea de autoridad se desdobla, con mayor o menor claridad y aclerto, pudiendo ocurrir: a) Que el contenido formal asignado a la segunda jefatura se limite a aspectos especificos de las actividades del escalón subordinado o a determinado tipo de problemas (fig. 2), en cuyo caso dicho órgano 
podrá quedar configurado como una jefatura funcional o b). Que su autoridad se extienda a toda clase de aspectos relativos a dichas actividades no reservados o asignados expresamente al primer jefe, en cuyo supuesto la segunda jefatura reviste el carácter de órgano

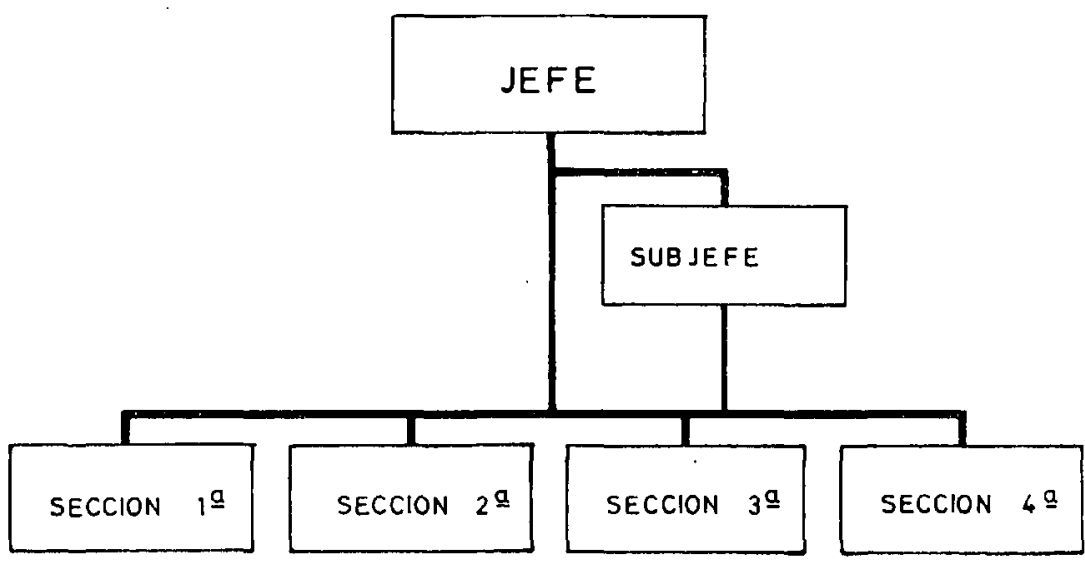

Figura 2

de línea, dado el contenido genérico del vínculo jerárquico (fig. 3). Estas modalidades, muy frecuentes en la práctica, no pueden ser objeto de una valoración de conjunto aplicable a las diversas situaciones

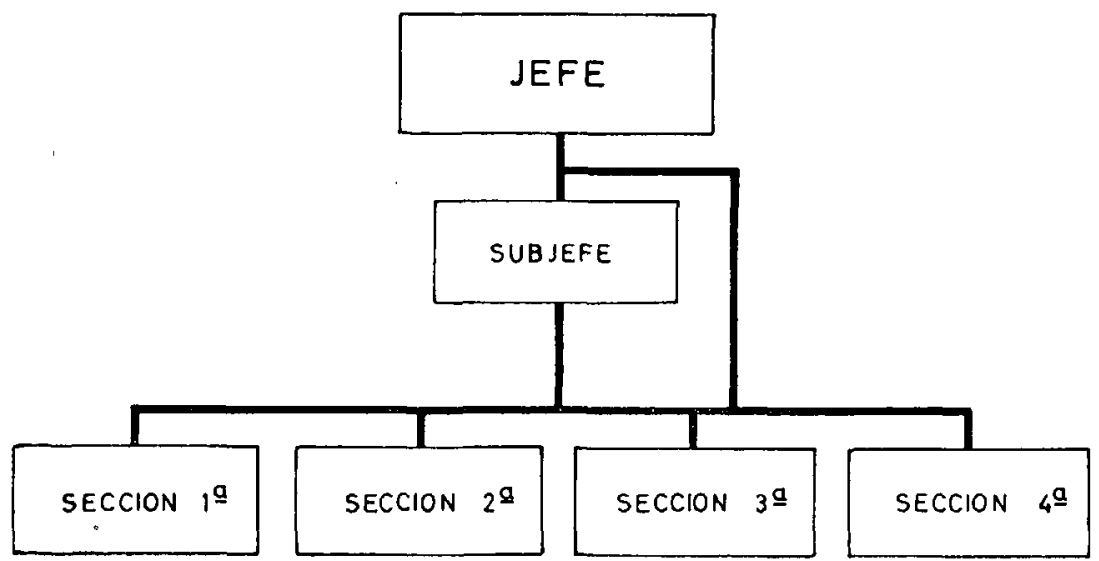

Figura 3 
DA-1967, núm. 109. LUIS BLANCO DE TELLA. Consideraciones en torno a la figura del segundo ...

concretas. Asi, por ejemplo, podrá darse el caso de que el superior retenga la casi totalidad de las funciones de dirección, quedando la segunda jefatura en posición directiva atenuada o prácticamente nominal (interposición débil). En este supuesto la figura del segundo jefe - aparte de las eventuales funciones de sustitución y representación del superior inmediato-queda caracterizada solamente por razón de su rango y el ejercicio de alguna facultad de dirección escasamente significativa.

A la inversa, cabe también que el segundo jefe, por la amplitud y variedad de las funciones directivas atribuidas al mismo, desempeñe. el papel fundamental en lo que se refiere a la dirección del escalón subordinado (interposición fuerte), aproximándose al supuesto comentado en el epigrafe anterior.

Algunas veces, especialmente cuando las unidades del escalón subordinado son excesivamente numerosas o constituyen una agrupación funcional heterogénea, la segunda jefatura parcialmente interpuesta puede dar buenos resultados prácticos siempre que se lleve a cabo entre ambas posiciones un reparto claro y equilibrado de funciones.

Pero si, por el contrario, el número de elementos del escalón inmediato inferior se mantiene dentro de limites razonables, agrupando funciones homogéneas y distribuidas según criterios uniformes, el segundo jefe parcialmente interpuesto puede resultar un elemento completamente innecesario.

Por otra parte, todos los casos de interposición parcial pueden plantear en la práctica delicados prồblemas de división de funciones entre las dos jefaturas consecutivas. La concurrencia directa de dos relaciones de autoridad formal sobre unos mismos elementos entraña siempre ciertos riesgos que no desaparecen por completo aunque dichas relaciones tengan su punto de arranque en órganos o posiclones jerarquizadas entre si.

\section{2,13 Interposición limitada a ciertas unidades del escalón subordinado}

En algunas ocasiones el segundo jefe aparece formalmente interpuesto en la línea de autoridad y responsabilidad solamente por lo que se refiere a una o varias unidades del escalón operativo, quedando las restantes bajo la dependencia inmediata del jefe superior y excluida, por tanto, en situaciones normales, de la autoridad de la segunda jefatura (Fig. 4).

Esta modalidad puede aparecer justificada por las mismas razo- 
DA-1967, núm. 109. LUIS BLANCO DE TELLA. Consideraciones en torno a la figura del segundo ...

nes expuestas en el punto anterior, es decir, cuando el nivel subordinado presenta un contenido funcional excesivamente complejo o un fraccionamiento orgánico que rebasa los límites prudentes en materia de amplitud del control. Por otra parte, resulta evidente que un reparto de unidades entre las dos jefaturas sucesivas provocará normalmente menos dificultades prácticas que un reparto de funciones sobre unas mismas unidades.

En la versión pura de esta variante la escala jerárquica presenta dos ramas de longitud desigual, como se indica en la figura 4. El jefe

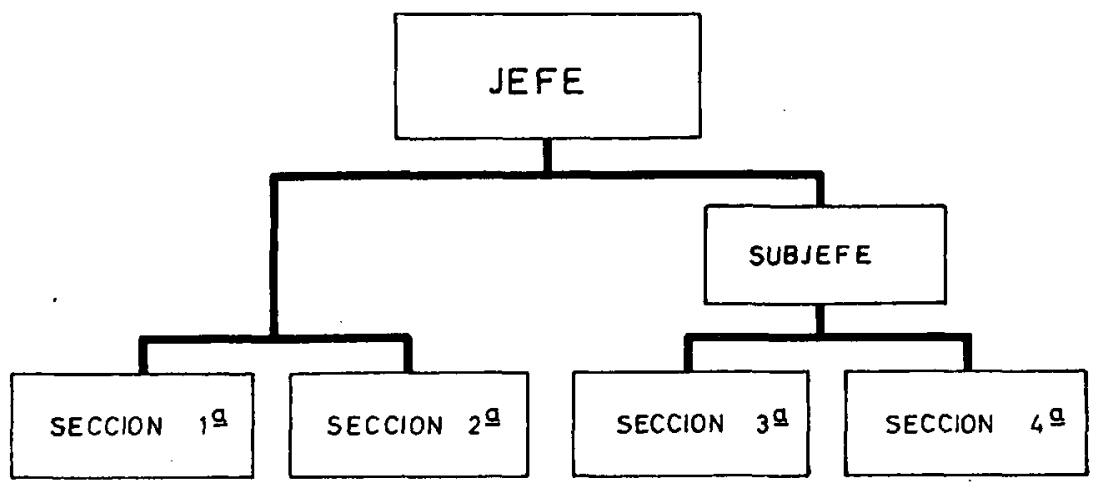

Figura 4

intermedio constituye un nivel orgánico más (jefatura de línea), pero sólo con relación a un sector concreto del escalón inferior. Las demás unidades encuadradas en dicho nivel quedan en este caso desligadas de la segunda jefatura.

En otros supuestos o derivaciones de esta modalidad, el órgano intermedio, aparte de aparecer configurado como jefatura de linea con relación a algunas unidades del escalón operativo, puede revestir además el carácter de jefatura funcional respecto a varias o a todas las restantes. Tal supuesto se representa en la figura 5.

\section{2,2 SEgundas Jefaturas IMPRopias}

Como tales consideramos las siguientes figuras que se caracterizan, como veremos a continuación, por la ausencia de alguno de los requisitos que hemos estimado esenciales en el epigrafe 1,1. 
DA-1967, núm. 109. LUIS BLANCO DE TELLA. Consideraciones en torno a la figura del segundo ...

Estudios

\section{2,21 Pluralidad de segundas jefaturas}

En determinadas organizaciones puede aparecer un escalón completo constituido por dos o más posiciones a las que se atribuye una denominación indicativa del carácter de segunda jefatura (varios subsecretarios, subdirectores, vicesecretarios, subdelegados, etc.) A nuestro juicio, la pluralidad de órganos de un mismo rango por de-

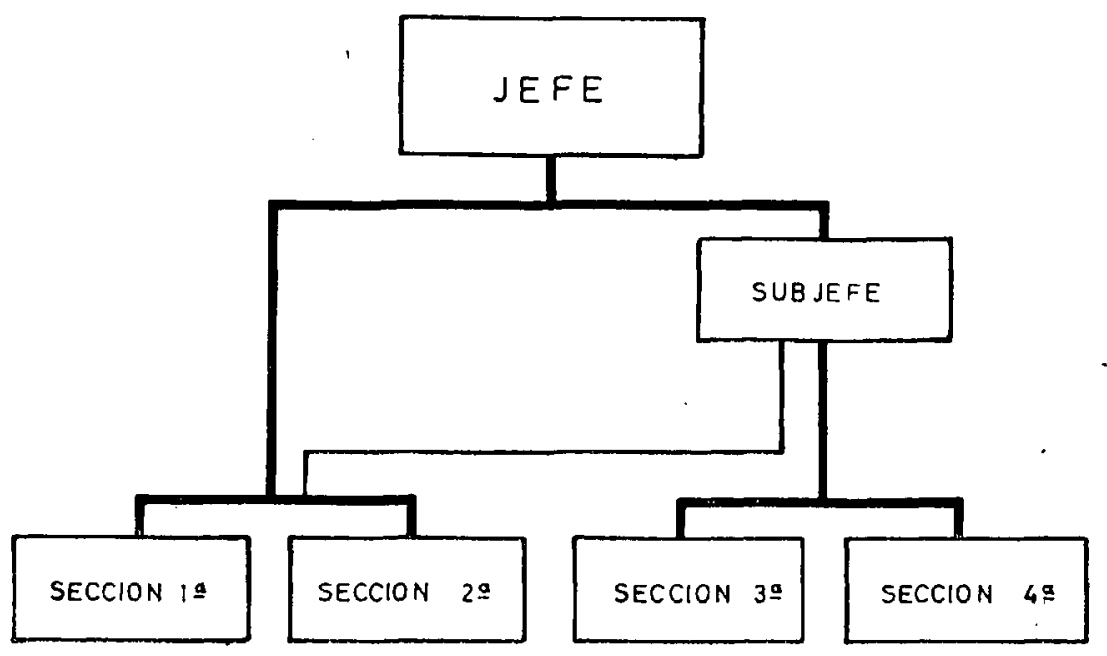

Figura 5

bajo del jefe superior equivale a la inexistencia o a la desaparición de la segunda jefatura. Tales posiciones, en realidad, son jefaturas de segundo nivel, lo cual resulta, evidentemente, bien distinto (figura 6).

El hecho de que alguno de los citados órganos aparezca en primer lugar en el orden de llamamientos a la sustitución y representación del superior 0 , incluso, tenga asignadas ciertas funciones generales o comunes cuyo ejercicio afecte a las unidades dependientes de los demás no altera, a nuestro modo de ver, el equilibrio horizontal del nivel de que se trata. Pensar otra cosa es confundir el concepto de rango con el de contenido funcional.

De ello resulta que los departamentos ministeriales dotados de dos o más subsecretarios carecen de segunda jefatura en sentido pro- 
DA-1967, núm. 109. LUIS BLANCO DE TELLA. Consideraciones en torno a la figura del segundo ...

pio, ya que a la vista del artículo 15-6 de la Ley de Régimen jurídico no puede sostenerse ninguna diferencia de rango, ni, mucho menos, la jerarquización entre los mismos. La misma conclusión cabe adoptar con relación a aquellas direcciones generales que cuentan con varios subdirectores.

En consecuencia, la existencia de varias segundas jefaturas encierra un problema semántico. La cuestión, en el fondo, se reduce

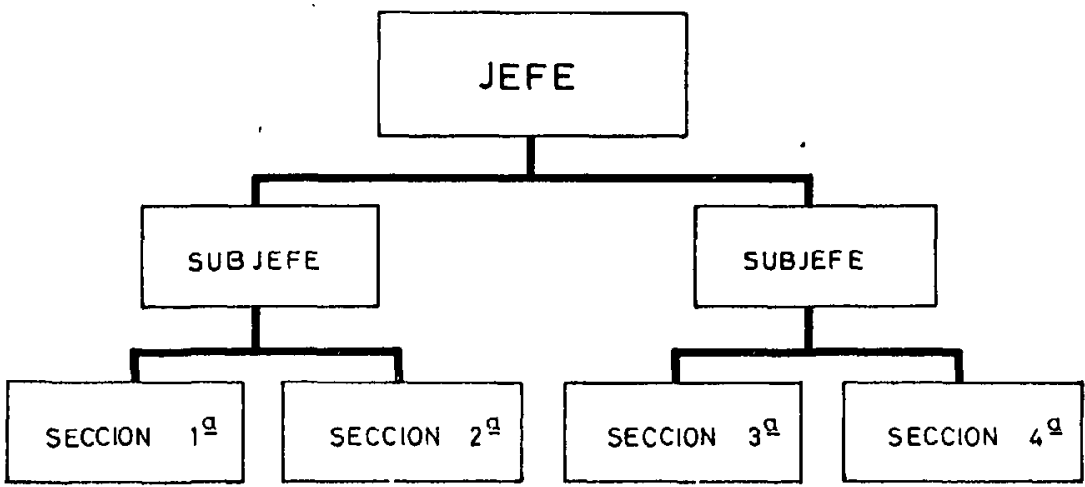

Figura 6

a determinar si el escalón integrado por dichas posiciones es necesario (cadena jerárquica corta) o innecesario (cadena jerárquica larga). La denominación que haya de darse a los elementos de dicho nivel constituye un aspecto secundario que queda al margen del presente estudio.

\section{2,22 Segunda jefatura configurada como órgano de apoyo}

Esta modalidad aparece cuando el denominado segundo jefe no ejerce, salvo caso de sustitución, autoridad directa sobre el escalón operativo, limitándose a prestar asistencia al jefe superior (Fig. 7). Su naturaleza es la de un órgano de apoyo o staff (normalmente de staff general), no constituyendo, por tanto, una segunda jefatura propiamente dicha a pesar de aparecer a veces designada como tal en consideración al rango que se le atribuye. Asi, por ejemplo, un vicesecretario general puede estar configurado como adjunto del secretario respectivo, sin tener bajo su dependencia directa a ninguna unidad o elemento de rango inferior. 
Esta variante impropia puede estar plenamente justificada en numerosas ocasiones, especialmente en niveles superiores donde las funciones directivas revisten su mayor complejidad. La posición de

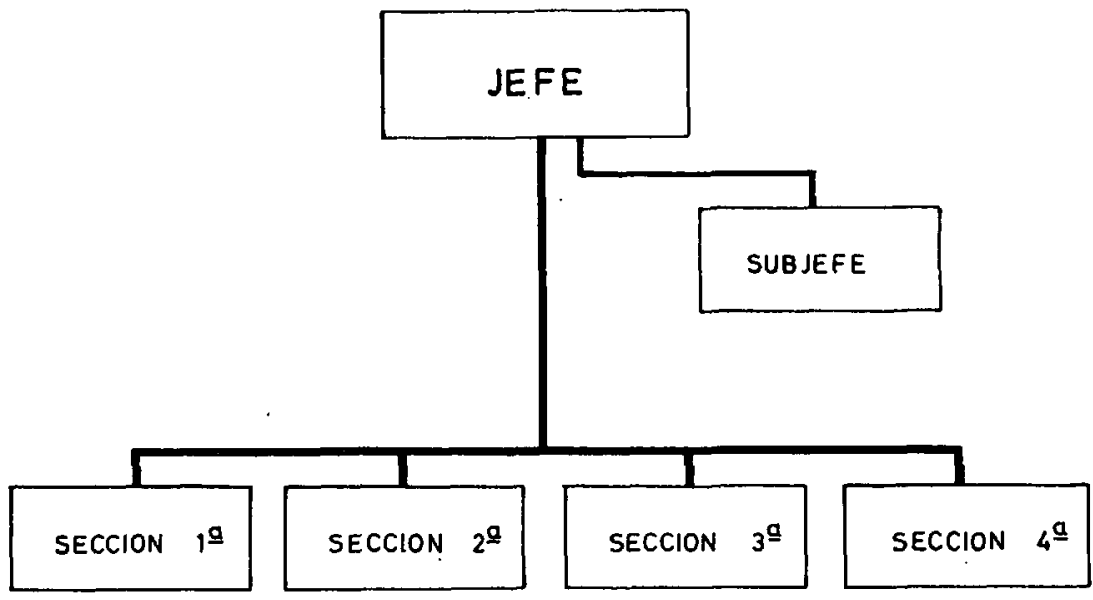

Figura 7

tales órganos con funciones de asistencia general ha de ser establecida formalmente con el mayor rigor posible, de forma que se limite su tendencia a asumir autoridad directa sobre el escalón inferior.

2,23 Segunda jefatura con funciones directivas sobre una agrupación de unidades de carácter instrumental

Una situación semejante en cierto modo a la anterior surge cuando al órgano calificado de segunda jefatura se le asigna la dirección de aquellas unidades encargadas de la prestación de funciones de carácter auxiliar (régimen interior, personal, suministros, archivo, etc.), careciendo de autoridad sobre los elementos de naturaleza operativa (figura 8). Dichos órganos, a los cuales se encomienda frecuentemente la prestación de una asistencia genérica al superior, constituyen en realidad jefaturas de servicios o departamentos de administración general. En ciertas ocasiones, las funciones instrumentales agrupadas normalmente bajo la autoridad de las citadas figuras se. incrementan con otras de tipo consultivo especializado (asesoría ju- 
DA-1967, núm. 109. LUIS BLANCO DE TELLA. Consideraciones en torno a la figura del segundo ...

rídica, asesoria financiera). Asi ocurre con determinadas secretarias generales a cuyo titular se atribuye reglamentariamente la denominación de segundo jefe del organismo o dependencia correspondiente.

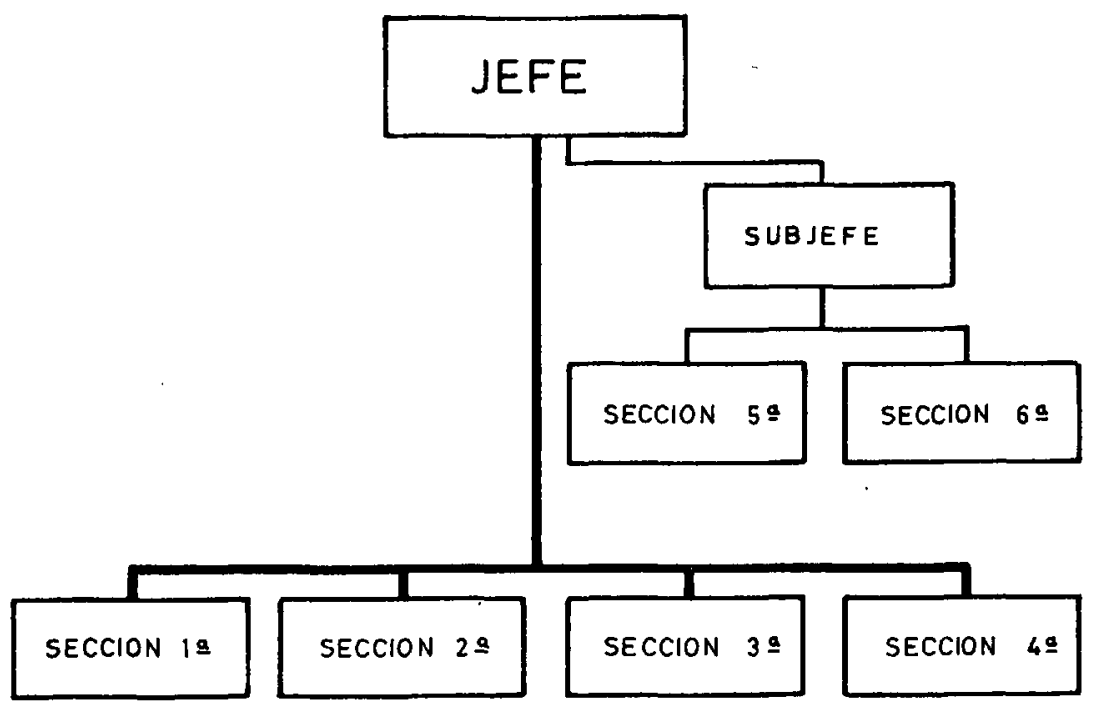

FIgURA 8

\section{Conclusión}

Los anteriores comentarios pueden servir, a nuestro juicio, para facilitar y aclarar el examen de las segundas jefaturas y demás elementos orgánicos semejantes existentes en la Administración, y que, a pesar de aparecèr designados muchas veces con idénticos términos, corresponden en realidad a posiciones radicalmente distintas o que, a la inversa, coinciden en su naturaleza y contenido funcional no obstante la diversidad de denominaciones.

Como observación final creemos conveniente hacer constar nuestra impresión de que una consideración detenida de las segundas jefaturas desde un plano racional debería provocar la supresión de muchas de ellas cuya existencia obedece a necesidades más aparentes que reales. No es posible, sin duda, formular un juicio de validez universal acerca de las citadas figuras, pero ante cada caso particular resulta fácil fundamentar una opinión razonable. 
Del mismo modo estimamos que debería frenarse la tendencia a establecer órganos de esta clase en los puntos más diversos, y a veces más inesperados, del aparato administrativo del Estado. A pesar de las trabas legales y reglamentarias con que tropieza la creación de nuevos órganos, este proceso resulta en la Administración bastante más expedito y libre de, resistencias que el que se encamina a su eliminación o supresión. 